\title{
High SLC17A9 expression correlates with poor survival in gastric carcinoma
}

\author{
Junqing $\mathrm{Li}^{\ddagger 1,2}$, Taiqiang $\mathrm{Su}^{\ddagger, 1,2}$, Liang Yang ${ }^{2,3}$, Lingna Deng ${ }^{4}$, Changhua Zhang ${ }^{1}$ \& Yulong \\ $\mathrm{He}^{*, 1,2}$ \\ ${ }^{1}$ Digestive Medicine Centre, Seventh Affiliated Hospital, Sun Yat-sen University, 628 Zhenyuan Road, Shenzhen 518000, PR China \\ 2Department of Gastrointestinal Surgery, First Affiliated Hospital, Sun Yat-sen University, 58 Zhongshan 2nd Road, Guangzhou \\ 510080, PR China \\ ${ }^{3}$ General Surgical Laboratory, First Affiliated Hospital, Sun Yat-sen University, Guangzhou 510080, PR China \\ ${ }^{4}$ Scientific Research Centre, Seventh Affiliated Hospital, Sun Yat-sen University, 628 Zhenyuan Road, Shenzhen 518000, PR China \\ *Author for correspondence: heyulong001@163.com \\ $\ddagger$ Authors contributed equally
}

\begin{abstract}
Aim: To elucidate the clinicopathological significance and prognostic value of SLC17A9 expression in gastric carcinoma (GC). Methods: SLC17A9 mRNA level and its relationship with TP53 mutation was analyzed. SLC17A9 protein expression was examined by immunohistochemistry in 161 patients. Results: SLC17A9 mRNA and protein expression were higher in GC tissues than in adjacent normal tissues $(p<0.01)$. SLC17A9 mRNA expression was higher in GC tissues having mutated TP53 than in tissues with wild-type TP53 ( $p<0.001$ ). High SLC17A9 expression was also significantly associated with poor overall survival and recurrence-free survival and was also found to be an independent prognostic factor for long-term survival in GC patients.Conclusion: Our results show that SLC17A9 may serve as a potential prognostic biomarker in GC patients.
\end{abstract}

First draft submitted: 16 May 2019; Accepted for publication: 18 September 2019; Published online: 4 December 2019

Keywords: gastric carcinoma $\bullet$ prognosis $\bullet$ SLC17A9 • TP53

Gastric carcinoma (GC) is one of the most common and lethal malignant cancers worldwide, especially in Eastern Asia [1,2]. GC imposes huge economic burdens on many families and countries [3]. Although advances in diagnosis and treatment have improved survival rates of patients with early GC [2], there is no effective treatment for patients with advanced GC, including targeted and immunotherapies [4]. Thus, a better understanding of the molecular mechanism of GC development may help us devise new therapeutic strategies and improve the prognosis of GC patients.

SLC17A9 is the fourth SLC17 gene type. It encodes a transmembrane protein involved in the transportation of small molecules [5]. As the first vesicular nucleotide transporter (VNUT), SLC17A9 participates in vesicular uptake, storage and secretion of nucleotides such as ATP and ADP [5-8]. SLC17A9 activity also plays an important role in maintaining lysosomal physiology and cell viability [9]. Recent studies indicate that overexpression of a short SLC17A9 transcript predicts poor survival in adult patients with T-cell acute lymphoblastic leukemia [10]. And SLC17A9 overexpression in colorectal cancer correlates with poor prognosis [11]. Although its specific role remains unclear, the above studies have suggested that SLC17A9 has a significant impact on cancer prognosis. But it is not clear whether high SLC17A9 expression correlates with poor survival in GC. Therefore, we conducted this study to quantify SLC17A9 expression in GC and normal tissues and then to assess its association with patients' prognoses.

First, the present study had evaluated the SLC17A9 mRNA level and its correlation with prognosis in GC using publicly available databases. Second, protein expression of SLC17A9 from our database had been analyzed using immunohistochemical (IHC) staining. Subsequently, correlations between SLC17A9 expression and clinicopathological parameters were analyzed, and the relationship between SLC17A9 and survival was determined. Furthermore, correlation between SLC17A9 mRNA expression and TP53 mutation was mined from publicly available databases, which might be a potential mechanism for SLC17A9 to promote GC formation. 
Publicly available databases utilized by the present study are widely used by medical researchers from all over the world, whose results regarding SCL17A9 have been confirmed by this study. Therefore, the findings of this research have practical implications and update our knowledge of the roles of SLC17A9.

\section{Materials \& methods}

Patient data

Data from 161 patients who received surgical treatment at the First Affiliated Hospital of Sun Yat-sen University (FAHSYSU) between 2004 and 2007 were chosen randomly and their follow-up data were retrospectively collected. Follow-ups were terminated by December 2017. None of the patients received chemotherapy or other treatments before sampling. Patients lost due to lack of compliance during follow-up or with incomplete data were excluded from this study. Clinicopathological characteristics of these patients included gender, age, tumor size, tumor location, Bornmann classification, differentiation, depth of invasion, lymph node metastasis, distant metastasis, clinical stage and carcinoembryonic antigen level. Patients were evaluated according to the recommendations made in the 8th edition of the American Joint Cancer Committee (AJCC) Tumor-Node-Metastasis (TNM) classifications. Another 61 patients who received surgical treatment in 2013 were chosen randomly for tissue microarrays.

Written informed consent was obtained from every patient. Ethical approval for clinical research was obtained from the Institutional Review Board of FAHSYSU.

\section{Tissue microarrays}

Paired adjacent normal and GC tissues of 61 patients were obtained from the Department of Pathology, FAHSYSU, and made into tissue microarrays (Servicebio, Wuhan, China) for IHC staining.

\section{IHC staining}

Paraffin-embedded GC tissues of 161 patients were obtained from the Department of Pathology, FAHSYSU. IHC staining of these GC tissues, and tissue microarrays, were conducted as described previously [11]. An anti-SLC17A9 antibody (1:200; EMD Millipore, MA, USA) was used for IHC staining.

Evaluation of the IHC-stained slides was performed by two independent investigators who were blinded to the specimens. Results were scored by a semiquantitative method [12]. First, no staining or less than $10 \%$ of the tumor cells stained was considered negative (IHC 0 ). Second, in $\geq 10 \%$ of tumor cells, IHC was scored using staining intensity as follows: 1 (weak), 2 (moderate) and 3 (strong). The last, scores of 0 and 1 were defined as low, and scores of 2 and 3 were defined as high SLC17A9 expression.

\section{Bioinformatics analysis}

RNASeq data for GC were downloaded from The Cancer Genome Atlas (TCGA) and Oncomine databases (https://www.oncomine.org/). The relationship between SLC17A9 mRNA expression and TP53 mutation was analyzed via LinkedOmics (http://www.linkedomics.org/). The prognostic value of the SLC17A9 mRNA level was analyzed via the Kaplan-Meier plot (http://www.kmplot.com/).

\section{Statistical analyses}

SPSS version 17.0 (IBM, IL, USA) was used for data analyses. The relationship between SLC17A9 expression and features of tumor progression was analyzed using the $\chi^{2}$ square test and Spearman correlation test. KaplanMeier survival curves were plotted, and the log-rank test was performed. Univariate and multivariate analyses were performed using the Cox proportional hazards model. The $\mathrm{p}<0.05$ was considered statistically significant.

\section{Results}

Higher SLC17A9 expression in GC tissues compared with normal gastric tissues

Data from TCGA were analyzed via UALCAN [13] (http://ualcan.path.uab.edu/). The results showed that the SLC17A9 gene was upregulated in GC tissues compared with normal tissues (Figure 1A). SLC17A9 mRNA levels in GC tissues $(\mathrm{n}=415)$ were higher than those in normal tissues $(\mathrm{n}=34 ; \mathrm{p}<0.001$, Figure 1B). The SLC17A9 mRNA level was also significantly upregulated with increasing tumor stage $(\mathrm{p}<0.001$, Figure $1 \mathrm{C})$. Analogous results were seen in the Oncomine cohorts (Table 1). We further assessed SLC17A9 protein expression by tissue microarrays with paired adjacent normal and GC tissues of 61 patients. Protein expression of SLC17A9 was 


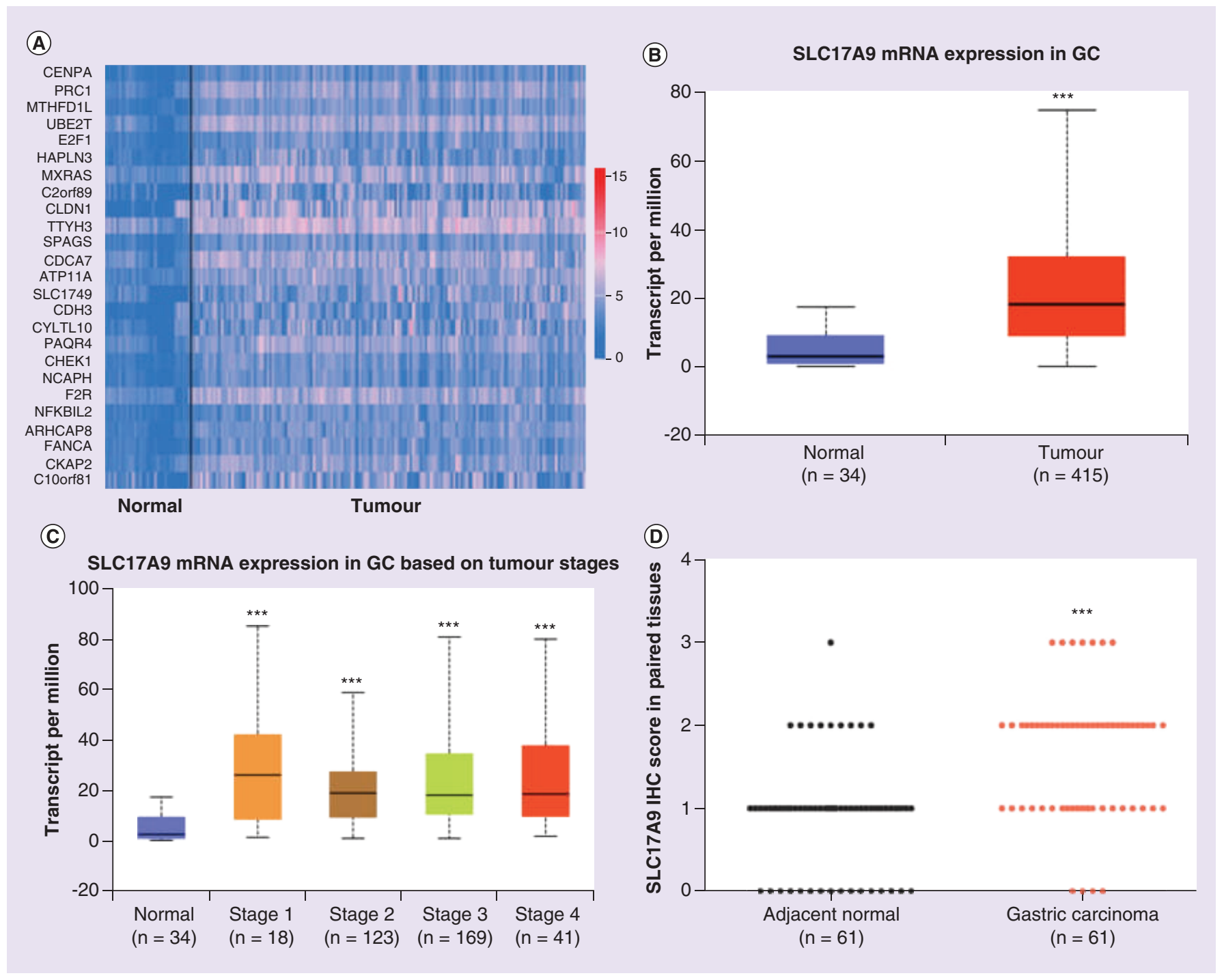

Figure 1. Upregulation of SLC17A9 in gastric carcinoma. (A) Analysis of TCGA data by a heat map. (B) SLC17A9 mRNA expression in GC and normal tissues from TCGA database. (C) SLC17A9 mRNA expression based on tumor stage from TCGA database. (D) Protein expression of SLC17A9 was significantly higher in GC tissues compared with adjacent normal tissues.

${ }^{* * *} p<0.001$.

GC: Gastric carcinoma; TCGA: The Cancer Genome Atlas.

significantly higher in GC tissues compared with adjacent normal tissues ( $\mathrm{p}<0.001$, Figure $1 \mathrm{D})$. Collectively, these data show that SLC17A9 expression was upregulated in GC tissues.

\section{Correlations between SLC17A9 expression \& clinicopathological parameters in GC}

To explore the association between SLC17A9 expression and clinicopathologic characteristics in GC, 161 paraffinembedded GC tissues were examined for SLC17A9 protein expression using IHC staining. SLC17A9 was expressed heterogeneously in GC tissues. As shown in Figure 2, the SLC17A9 proteins of most patients with GC were located in the cytoplasms and cytomembranes of tumor cells. According to SLC17A9 protein expression in GC tissues as indicated by IHC scoring, there were 24 cases (14.9\%) with an IHC score of 0, 52 (32.3\%) with an IHC score of 1, 57(35.4\%) with an IHC score of 2 and 28 (17.4\%) with an IHC score of 3.

The association between SLC17A9 expression and clinicopathological variables was determined by the $\chi^{2}$ square test and Spearman correlation test (Table 2). The constituent ratio between low and high SLC17A9 expression groups was significantly different in tumor size $(\mathrm{p}<0.05)$, depth of invasion $(\mathrm{p}<0.05)$, lymph node metastasis 


\begin{tabular}{|c|c|c|c|c|c|}
\hline Cohort & Sample (n) & t-test & Fold change & p-value & Ref. \\
\hline Cui et al. & Gastric cancer (80) vs normal (80) & 2.003 & 1.204 & $0.023^{*}$ & [14] \\
\hline \multirow[t]{5}{*}{ Deng et al. } & Diffuse gastric adenocarcinoma (36) vs normal (98) & 4.275 & 1.041 & $0.000 * * *$ & [15] \\
\hline & Gastric adenocarcinoma (63) vs normal (98) & 4.574 & 1.072 & $0.000 * * *$ & \\
\hline & Gastric cancer (42) vs normal (98) & 5.049 & 1.072 & $0.000 * * *$ & \\
\hline & Gastric intestinal type adenocarcinoma (38) vs normal (98) & 5.879 & 1.109 & $0.000 * * *$ & \\
\hline & Gastric mixed adenocarcinoma (11) vs normal (98) & 2.990 & 1.127 & $0.007 * *$ & \\
\hline \multirow[t]{5}{*}{ TCGA } & Diffuse gastric adenocarcinoma (45) vs normal (94) & 4.885 & 1.096 & $0.000 * * *$ & \\
\hline & Gastric adenocarcinoma (173) vs normal (94) & 10.689 & 1.186 & $0.000 * * *$ & \\
\hline & Gastric intestinal type adenocarcinoma (41) vs normal (41) & 6.560 & 1.261 & $0.000 * * *$ & \\
\hline & Gastric tubular adenocarcinoma (31) vs normal (94) & 6.601 & 1.256 & $0.000 * * *$ & \\
\hline & Mucinous gastric adenocarcinoma (12) vs normal (94) & 4.003 & 1.189 & $0.001 * *$ & \\
\hline Wang et al. & Gastric cancer (12) vs normal (17) & 1.765 & 1.439 & $0.047^{*}$ & [16] \\
\hline
\end{tabular}

$(\mathrm{p}<0.01)$ and AJCC stage $(\mathrm{p}<0.01)$. SLC17A9 expression in GC tissues was significantly correlated with tumor size $(\mathrm{p}<0.05)$, lymph node metastasis $(\mathrm{p}<0.01)$ and AJCC cTNM stage $(\mathrm{p}<0.01)$.

\section{SLC17A9 overexpression was associated with poor survival in GC}

To determine the prognostic value of SLC17A9 expression in GC, we first analyzed the data via the Kaplan-Meier plot [17]. Patients with a high SLC17A9 mRNA level had worse overall survival (OS) (Figure 3A) and recurrencefree survival (RFS) (Figure 3B). Second, the prognostic role of SLC17A9 protein expression was assessed by a Kaplan-Meier method. The follow-up period of the included patients ranged between 2 months and 156 months and the mean survival time of them was $63.5 \pm 4.6$ months. The mean survival of patients with low expression of SLC17A9 was $82.8 \pm 7.0$ months while it was $45.5 \pm 5.2$ months for those patients with high SLC17A9 expression. The 5-year OS rate of all patients was 37.1, and 52.1\% in the SLC17A9 low and $23.5 \%$ in the high SLC17A9 expression group. These data indicated that patients with high SLC17A9 expression had worse OS (Figure 3C) and RFS (Figure 3D) (both $\mathrm{p}<0.001$ ). Moreover, we determined the prognostic value of SLC17A9 expression in early (cTNM I and II) and advanced (cTNM III and IV) GC. The results showed that patients with high SLC17A9 expression had worse OS (Figure 3E) and RFS (Figure 3F) (both $p<0.01$ ) in early-stage GC. Low SLC17A9 expression was not associated with better OS $(p=0.074$, Figure $3 \mathrm{G})$ but was associated with better RFS $(\mathrm{p}<0.05$, Figure $3 \mathrm{H})$ in advanced GC.

\section{SLC17A9 expression was an independent prognostic factor for GC patient}

The effects of SLC17A9 expression and other clinicopathological variables on GC patients' prognoses were evaluated by univariate and multivariate analysis. The detailed results of the univariate analysis were demonstrated in Tables 3 and 4. The multivariate Cox regression analysis demonstrated SLC17A9 expression as an independent predictor of OS ( $\mathrm{p}<0.05$; Table 3) and RFS ( $p<0.01$; Table 4) in GC patients. In summary, we proved that SLC17A9 expression could serve as a molecular marker to independently predict GC patients' prognoses.

The relationship between SLC17A9 mRNA expression \& TP53 mutation in GC

LinkedOmics data [18] showed that SLC17A9 mRNA expression was significantly associated with many gene mutations. As shown in Figure 4A, the expression of SLC17A9 mRNA correlated positively with mutated TP53. SLC17A9 mRNA levels in GC tissues with mutated TP53 were higher than those with wild-type TP53 $(\mathrm{p}<0.001$, Figure 4B). These results were from publicly available databases which were real world databases and were widely used in medical research. So, these results had practical implications.

\section{Discussion}

Our study demonstrates that high SLC17A9 expression is significantly associated with poor prognosis and its expression may serve as a biomarker for the prognosis of patients with GC. First, we assessed the SLC17A9 mRNA level from data in the TCGA and Oncomine database. The results indicated that the SLC17A9 mRNA level was 
Table 2. Correlations between SLC17A9 expression and clinicopathologic parameters of 161 gastric carcinoma

\section{patients.}

\begin{tabular}{|c|c|c|c|c|c|c|c|}
\hline \multirow[t]{2}{*}{ Characteristics } & \multirow[t]{2}{*}{$\mathbf{n}$} & \multicolumn{2}{|c|}{ SLC17A9 Expression } & \multirow[t]{2}{*}{$x^{2}$} & \multirow[t]{2}{*}{ p-value } & \multirow[t]{2}{*}{$\rho^{* * *}$} & \multirow[t]{2}{*}{ p-value } \\
\hline & & Low $(n=76)$ & High $(n=85)$ & & & & \\
\hline Age & & $57.75 \pm 12.17$ & $57.89 \pm 11.86$ & & & & \\
\hline$<60$ years & $85(52.8 \%)$ & $43(26.7 \%)$ & $42(26.1 \%)$ & 0.827 & 0.363 & 0.072 & 0.366 \\
\hline$\geq 60$ years & $76(47.2 \%)$ & $33(20.5 \%)$ & $43(26.7 \%)$ & & & & \\
\hline \multicolumn{8}{|l|}{ Gender } \\
\hline Males & $105(65.2 \%)$ & $49(30.4 \%)$ & $56(34.8 \%)$ & 0.035 & 0.851 & -0.015 & 0.853 \\
\hline Females & $56(34.8 \%)$ & $27(16.8 \%)$ & $29(18.0 \%)$ & & & & \\
\hline \multicolumn{8}{|l|}{ Tumor location } \\
\hline Proximal & $35(21.7 \%)$ & $17(10.6 \%)$ & $18(11.2 \%)$ & 2.118 & 0.548 & 0.071 & 0.368 \\
\hline Middle & $31(19.3 \%)$ & $18(11.2 \%)$ & $13(8.1 \%)$ & & & & \\
\hline Distal & $58(36.0 \%)$ & $25(15.5 \%)$ & $33(20.5 \%)$ & & & & \\
\hline More than 2 & $37(23.0 \%)$ & $16(9.9 \%)$ & $21(13.0 \%)$ & & & & \\
\hline \multicolumn{8}{|l|}{ Tumor size } \\
\hline$<5 \mathrm{~cm}$ & $74(46.0 \%)$ & $43(26.7 \%)$ & $31(19.3 \%)$ & 6.532 & $0.011 *$ & 0.201 & $0.01 *$ \\
\hline$\geq 5 \mathrm{~cm}$ & $87(54.0 \%)$ & $33(20.5 \%)$ & $54(33.5 \%)$ & & & & \\
\hline \multicolumn{8}{|l|}{ Histologic type } \\
\hline Other adenocarcinoma & $137(85.1 \%)$ & $66(41.0 \%)$ & $71(44.1 \%)$ & 1.777 & 0.620 & 0.043 & 0.590 \\
\hline Signet ring & $10(8.1 \%)$ & $4(3.1 \%)$ & $6(5.0 \%)$ & & & & \\
\hline Mucinous & $13(6.2 \%)$ & $5(2.5 \%)$ & $8(3.7 \%)$ & & & & \\
\hline Undifferentiated & $1(0.6 \%)$ & $1(0.6 \%)$ & $0(0)$ & & & & \\
\hline \multicolumn{8}{|l|}{ Bornmann classification } \\
\hline 1 & $8(5.0 \%)$ & $3(1.9 \%)$ & $5(3.1 \%)$ & 7.541 & 0.057 & 0.064 & 0.421 \\
\hline II & $32(19.9 \%)$ & $21(13.0 \%)$ & $11(6.8 \%)$ & & & & \\
\hline III & $102(63.4 \%)$ & $41(25.5 \%)$ & $61(37.9 \%)$ & & & & \\
\hline IV & $19(11.8 \%)$ & $11(6.8 \%)$ & $8(5.0 \%)$ & & & & \\
\hline \multicolumn{8}{|l|}{ Differentiation } \\
\hline Well & $4(2.5 \%)$ & $3(1.9 \%)$ & $1(0.6 \%)$ & 1.502 & 0.472 & 0.067 & 0.397 \\
\hline Moderate & $36(22.4 \%)$ & $18(11.2 \%)$ & $18(11.2 \%)$ & & & & \\
\hline Poor & $121(75.2 \%)$ & $55(34.2 \%)$ & $66(41.0 \%)$ & & & & \\
\hline \multicolumn{8}{|l|}{ Depth of invasion } \\
\hline $\mathrm{T} 1$ & $15(9.3 \%)$ & $8(5.0 \%)$ & $7(4.3 \%)$ & 11.032 & $0.012^{*}$ & 0.153 & 0.053 \\
\hline $\mathrm{T} 2$ & $15(9.3 \%)$ & $13(8.1 \%)$ & $2(1.2 \%)$ & & & & \\
\hline T3 & $88(54.7 \%)$ & $37(23.0 \%)$ & $51(31.7 \%)$ & & & & \\
\hline $\mathrm{T} 4$ & $43(26.7 \%)$ & $18(11.2 \%)$ & $25(15.5 \%)$ & & & & \\
\hline \multicolumn{8}{|l|}{ Lymph node metastasis } \\
\hline No & $50(31.1 \%)$ & $33(20.5 \%)$ & $17(10.6 \%)$ & 12.617 & $0.006 * *$ & 0.225 & $0.004 * *$ \\
\hline N1 & $51(31.7 \%)$ & $22(13.7 \%)$ & $29(18.0 \%)$ & & & & \\
\hline $\mathrm{N} 2$ & $24(14.9 \%)$ & $6(3.7 \%)$ & $18(11.2 \%)$ & & & & \\
\hline N3 & $36(22.4 \%)$ & $15(9.3 \%)$ & $21(13.0 \%)$ & & & & \\
\hline \multicolumn{8}{|l|}{ Distant metastases } \\
\hline Mo & $129(80.1 \%)$ & $65(40.4 \%)$ & $64(39.8 \%)$ & 2.638 & 0.104 & 0.128 & 0.106 \\
\hline M1 & $32(19.9 \%)$ & $11(6.8 \%)$ & $21(13.0 \%)$ & & & & \\
\hline \multicolumn{8}{|l|}{ AJCC CTNM } \\
\hline $\mathrm{I}+\mathrm{II}$ & $53(32.9 \%)$ & $35(21.7 \%)$ & $18(11.2 \%)$ & 12.244 & $0.001 * *$ & 0.264 & 0.001 ** \\
\hline III+IV & $108(67.1 \%)$ & $41(25.5 \%)$ & $67(41.6 \%)$ & & & & \\
\hline \multicolumn{8}{|l|}{ CEA level $(\mu \mathrm{g} / \mathrm{l})$} \\
\hline$<5$ & $142(88.2 \%)$ & $69(42.9 \%)$ & $73(45.3 \%)$ & 0.928 & 0.335 & 0.076 & 0.338 \\
\hline$\geq 5$ & $19(11.8 \%)$ & $7(4.3 \%)$ & $12(7.5 \%)$ & & & & \\
\hline
\end{tabular}




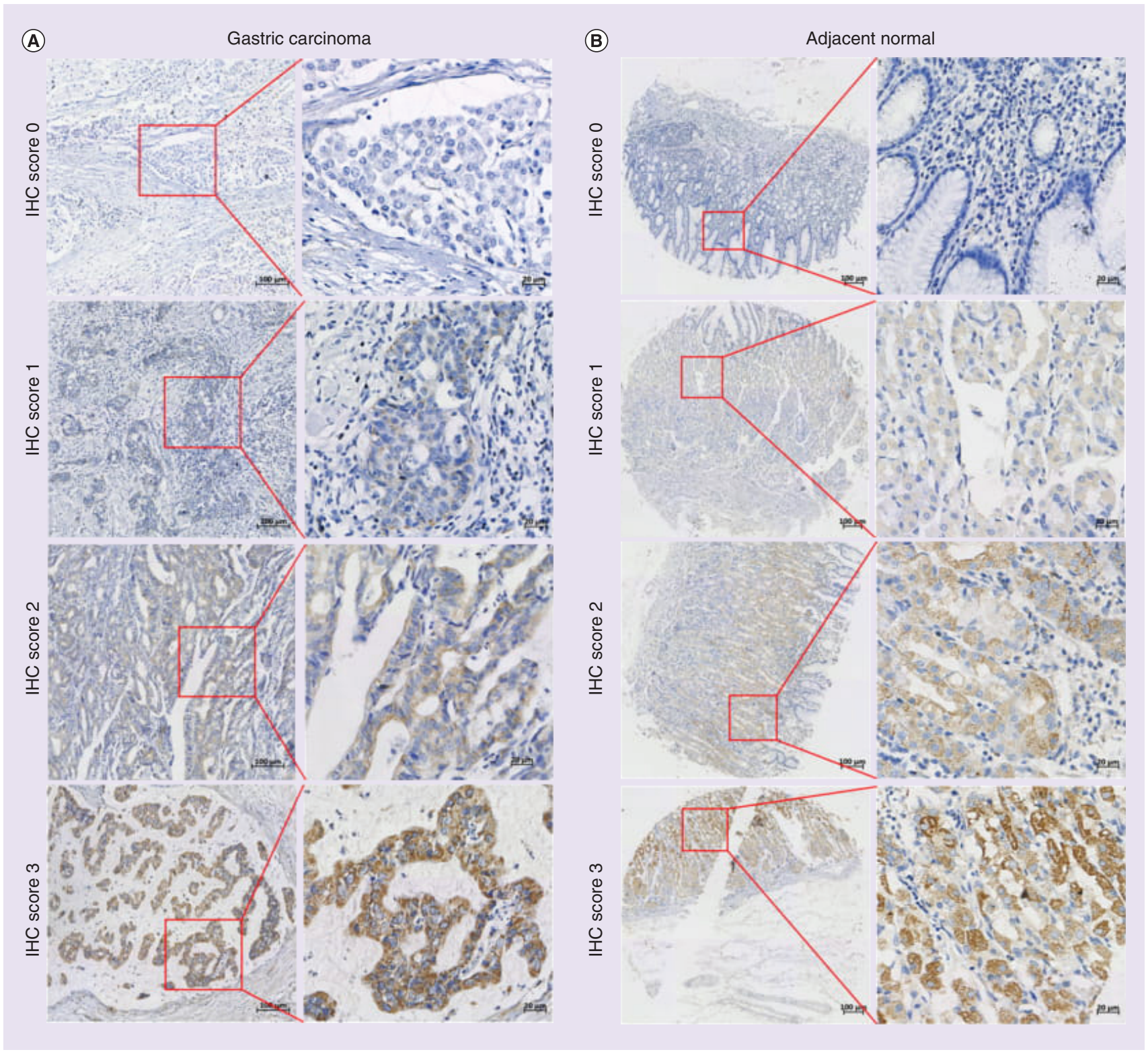

Figure 2. Immunohistochemical analysis of SLC17A9 protein expression in gastric carcinoma and adjacent normal tissues. IHC staining of (A) the SLC17A9 protein in GC tissues and (B) adjacent normal tissues.

GC: Gastric carcinoma; IHC: Immunohistochemistry.

significantly higher in GC than in normal tissues. Interestingly, the expression of SLC17A9 mRNA correlated positively with TP53 mutation. Second, we explored whether the expression of SLC17A9 mRNA was related to a poor prognosis. The results from the Kaplan-Meier plot showed that patients with high SLC17A9 mRNA expression had worse OS and RFS. Third, tissue microarrays for 61 patients showed that SLC17A9 protein expression was significantly higher in GC than in adjacent normal tissues. Furthermore, we performed IHC staining and analyzed the relationship between SLC17A9 expression and prognosis in $161 \mathrm{GC}$ patients. The results were the same as that of the bioinformatics analysis. Finally, the multivariate Cox regression analysis demonstrated SLC17A9 expression as an independent prognostic factor of both OS and RFS in GC patients.

SLC17A9 is the fourth SLC17 gene [5]. These genes are expressed widely in various organs such as the brain, and adrenal and thyroid glands [8]. The SLC17A9 protein is a VNUT that participates in transporting nucleotides [8]. As a VNUT, the SLC17A9 protein plays an important role in transporting ATP in airway neutrophils as well as 
(A)
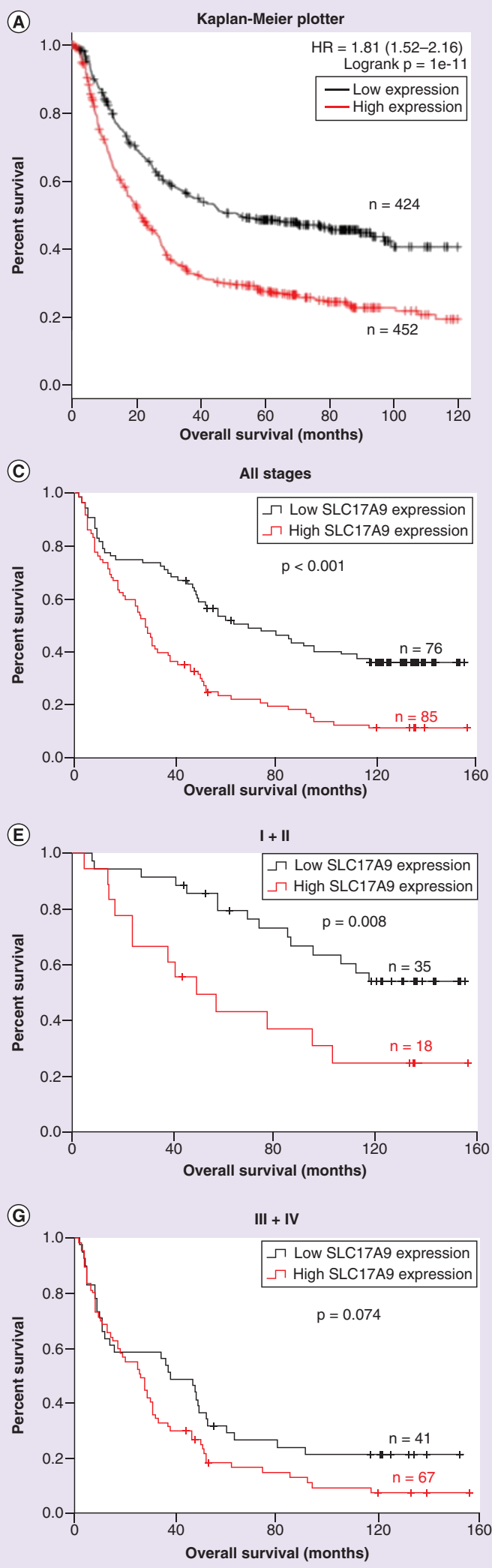

(B)
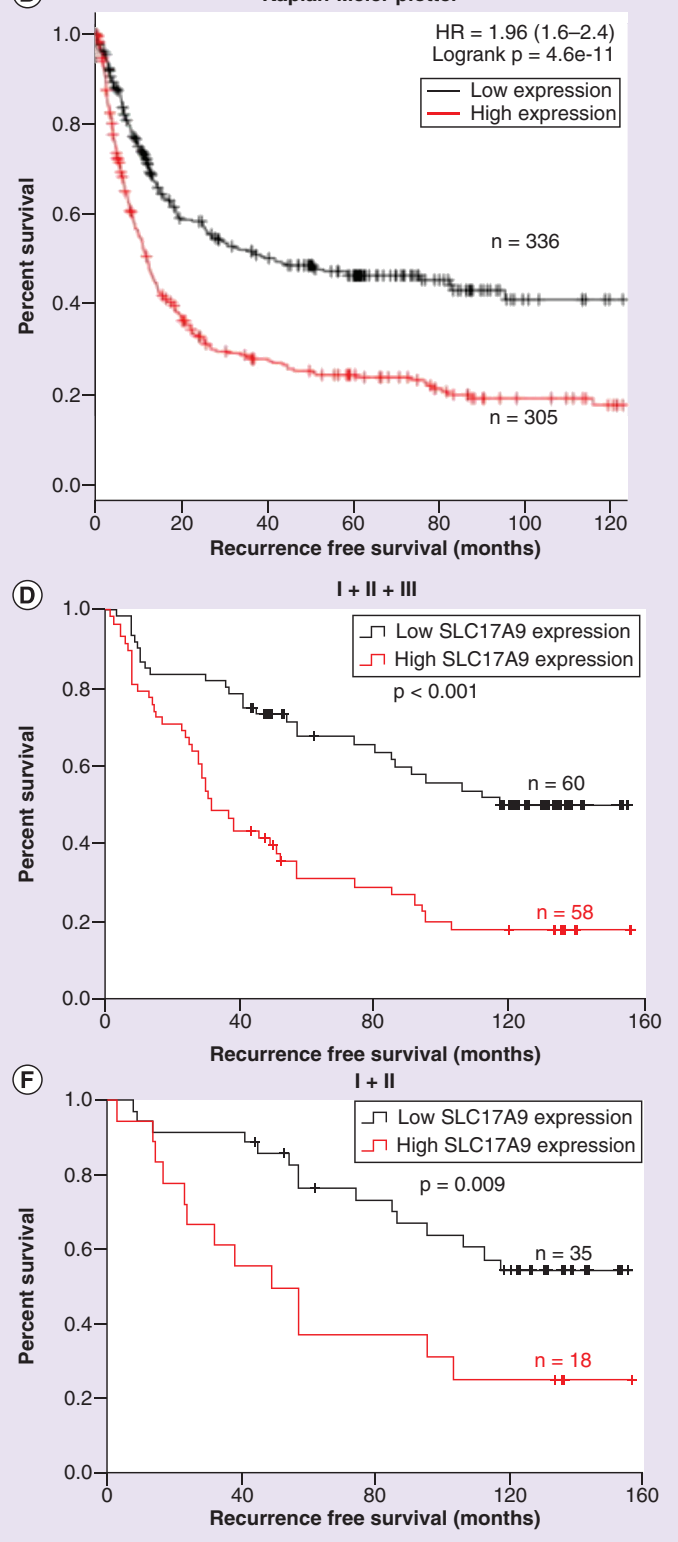

(H)

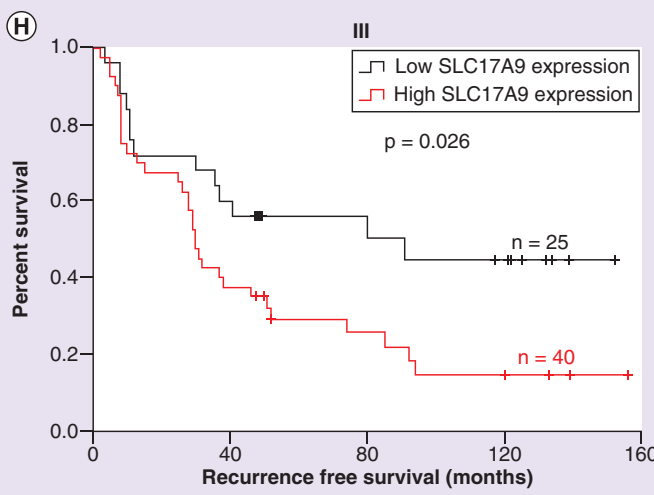

Figure 3. Gastric carcinoma patients overexpressing SLC17A9 have a poor prognosis. Kaplan-Meier plotter (A) OS and (B) RFS curves for GC patients with low and high SLC17A9 mRNA levels. GC patients with high SLC17A9 expression have worse (C) OS and (D) RFS than those with low SLC17A9 expression. Patients with high SLC17A9 expression have worse (E) OS and (F) RFS in early GC (cTNM I and II). (G) There is no significant difference in OS between the high and low SLC17A9 expression groups. (H) Patients with high SLC17A9 expression have worse RFS in advanced GC (cTNM III).

CTNM: clinical tumor-node-metastasis; GC: Gastric carcinoma; HR: Hazard ratio; OS: Overall survival; RFS: Recurrence-free survival. 


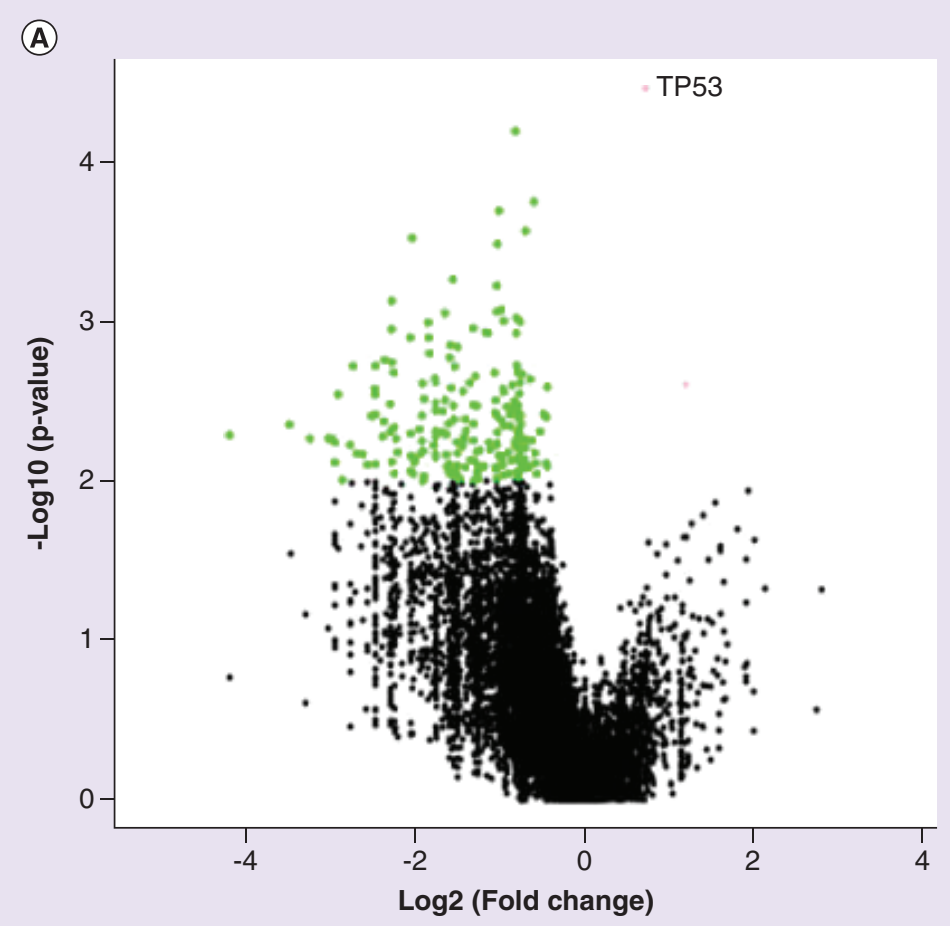

(B)

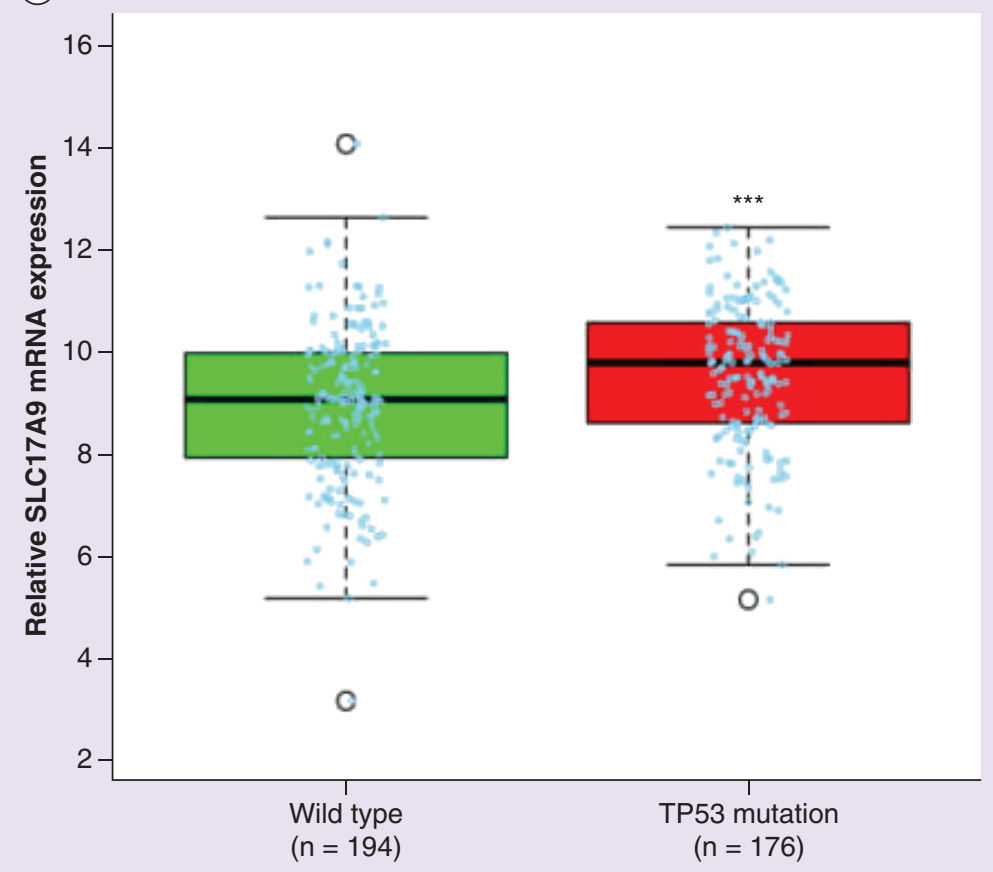

Figure 4. Relationship between SLC17A9 mRNA expression and TP53 mutation. (A) Volcano plot of the relationship between SLC17A9 mRNA expression and multiple gene mutations. (B) SLC17A9 mRNA expression in wild-type and mutant TP53 GC.

${ }^{* * *} \mathrm{p}<0.001$.

GC: Gastric carcinoma. 
Table 3. Cox proportional-hazard regression analysis for overall survival.

\begin{tabular}{|c|c|c|c|c|c|c|c|c|}
\hline \multirow[t]{3}{*}{ Characteristic } & \multicolumn{4}{|c|}{ Univariate analysis } & \multicolumn{4}{|c|}{ Multivariate analysis } \\
\hline & \multirow[t]{2}{*}{$p$-value } & \multirow[t]{2}{*}{ HR } & \multicolumn{2}{|c|}{$95.0 \% \mathrm{Cl}$ for $\operatorname{Exp}(\mathrm{B})$} & \multirow[t]{2}{*}{ p-value } & \multirow[t]{2}{*}{ HR } & \multicolumn{2}{|c|}{$95.0 \% \mathrm{Cl}$ for $\operatorname{Exp}(\mathrm{B})$} \\
\hline & & & Lower & Upper & & & Lower & Upper \\
\hline Gender & $0.022^{*}$ & 1.544 & 1.064 & 2.241 & & & & \\
\hline Age & 0.268 & 0.816 & 0.570 & 1.169 & & & & \\
\hline Tumor location & 0.730 & 1.032 & 0.865 & 1.230 & & & & \\
\hline Tumor size & $0.000 * * *$ & 2.088 & 1.442 & 3.025 & & & & \\
\hline Histologic type & 0.658 & 1.043 & 0.865 & 1.258 & & & & \\
\hline Bornmann classification & $0.006^{* *}$ & 1.465 & 1.117 & 1.922 & $0.023^{*}$ & 1.453 & 1.052 & 2.008 \\
\hline Differentiation & $0.011 *$ & 1.682 & 1.125 & 2.516 & & & & \\
\hline Depth of invasion & $0.000 * * *$ & 1.750 & 1.378 & 2.222 & & & & \\
\hline Lymph node metastasis & $0.000 * * *$ & 1.498 & 1.280 & 1.753 & & & & \\
\hline Distant metastases & $0.000 * * *$ & 3.763 & 2.466 & 5.742 & & & & \\
\hline AJCC CTNM & $0.000 * * *$ & 2.086 & 1.675 & 2.599 & & & & \\
\hline CEA & $0.028^{*}$ & 1.786 & 1.066 & 2.993 & & & & \\
\hline SLC17A9 expression & $0.000 * * *$ & 2.163 & 1.490 & 3.141 & $0.021 *$ & 1.622 & 1.077 & 2.444 \\
\hline
\end{tabular}

Table 4. Cox proportional-hazard regression analysis for recurrence free survival.

\begin{tabular}{|c|c|c|c|c|c|c|c|c|}
\hline \multirow[t]{3}{*}{ Characteristic } & \multicolumn{4}{|c|}{ Univariate analysis } & \multicolumn{4}{|c|}{ Multivariate analysis } \\
\hline & \multirow[t]{2}{*}{ p-value } & \multirow[t]{2}{*}{ HR } & \multicolumn{2}{|c|}{$95.0 \% \mathrm{Cl}$ for $\operatorname{Exp}(\mathrm{B})$} & \multirow[t]{2}{*}{ p-value } & \multirow[t]{2}{*}{ HR } & \multicolumn{2}{|c|}{$95.0 \% \mathrm{Cl}$ for $\operatorname{Exp}(\mathrm{B})$} \\
\hline & & & Lower & Upper & & & Lower & Upper \\
\hline Gender & 0.126 & 1.461 & 0.899 & 2.375 & & & & \\
\hline Age & 0.974 & 0.992 & 0.627 & 1.571 & & & & \\
\hline Tumor location & 0.639 & 0.948 & 0.759 & 1.184 & & & & \\
\hline Tumor size & $0.019 *$ & 1.740 & 1.097 & 2.760 & & & & \\
\hline Histologic type & 0.585 & 1.068 & 0.844 & 1.351 & & & & \\
\hline Bornmann classification & $0.003 * *$ & 1.716 & 1.207 & 2.440 & $0.005 * *$ & 1.847 & 1.208 & 2.822 \\
\hline Differentiation & 0.053 & 1.606 & 0.993 & 2.595 & & & & \\
\hline Depth of invasion & $0.006 * *$ & 1.483 & 1.118 & 1.968 & & & & \\
\hline Lymph node metastasis & 0.057 & 1.241 & 0.993 & 1.551 & & & & \\
\hline AJCC CTNM & $0.001 * *$ & 1.737 & 1.251 & 2.412 & & & & \\
\hline CEA & 0.328 & 1.476 & 0.676 & 3.220 & & & & \\
\hline SLC17A9 expression & $0.000 * * *$ & 2.618 & 1.622 & 4.227 & $0.001 * *$ & 2.348 & 1.393 & 3.958 \\
\hline
\end{tabular}

epithelial, astrocyte, adrenal chromaffin and pancreatic cells [6-8,19,20]. Cao et al. [9] have shown that SLC17A9 functions as a lysosomal ATP transporter and maintains cell viability.

These studies indicate that the SLC17A9 protein plays an important physiological role in many cells. Conversely, abnormal expression of SLC17A9 can lead to disease. For example, functional abnormalities of the SLC17A9 protein are related to disseminated superficial actinic porokeratosis [21]. An aberrantly overexpressed short mRNA transcript of the $S L C 17 A 9$ gene is present in most cases of T-cell acute lymphoblastic leukemia, predicting a poor prognosis in adults [10]. Some studies revealed that SLC17A9-deficient cells exhibited lysosome dysfunction and underwent cell death [9]. Furthermore, SLC17A9 knockdown can decrease cell migration in human lung cancer cells [22]. A recent study indicated that SLC17A9 overexpression in colorectal cancer correlated with poor prognosis [11]. It was proven that high SLC17A9 expression heralded poor prognoses of GC patients which deepened our understanding of the initiation and progression of GC.

We also found that high expression of SLC17A9 in GC tissues was related to a greater tumor size, depth of invasion, lymph node metastasis and AJCC cTNM stage. However, the impact of SLC17A9 on GC, and 
its mechanisms, was not examined, which is a limitation of this study. The SLC17A9 protein functions as an ATP transporter and regulates cell viability [11]. Because ATP is involved in various tumor cell metabolic processes, especially glycolysis [23] that is critical for sustaining cancer cell proliferation and aggressiveness [24,25], we extrapolate that upregulating SLC17A9 expression promotes the proliferation and invasion abilities of GC cells by enhancing glycolysis.

The SLC17A9 protein is associated with cell migration, and SLC17A9 knockdown decreases cell migration in human lung cancer cells [22]. The VNUT is responsible for vesicular ATP release and neutrophil migration [20]. Also, microglial migration is mediated by ATP release from lysosomes [26]. Based on these findings, we infer that SLC17A9 overexpression may increase the metastatic ability of GC cells. The above studies indicated that SLC17A9 played an important role in the storage, transportation and release of ATP. Moreover, as a post-translational modification, ubiquitination is an ATP-dependent process sequentially mediated by three enzymes $[27,28]$ and supports aberrant oncogenic signaling for survival and metastasis [29]. According to these studies, SLC17A9 may affect tumor cell survival by modulating ubiquitination. Importantly, we discovered that SLC17A9 mRNA expression correlated positively with TP53 mutation in GC. TP53 is a tumor suppressor gene and its mutation plays an important role in the development and progression of many cancers, including GC [30]. And P53 degradation is regulated via the ubiquitin-proteasome system [27]. Therefore, it is worth for further research to explore the mechanism of SLC17A9 in GC and its relationship with TP53 mutations.

\section{Conclusion}

Taken together, our results show that high SLC17A9 expression indicates a poor prognosis in GC patients. Thus, SLC17A9 might serve as an independent prognostic factor for GC patients.

\section{Summary points}

- SLC17A9 was overexpressed in gastric carcinoma (GC) tissues compared with adjacent normal tissues.

- High SLC17A9 expression indicates poor overall survival and recurrence-free survival in GC patients.

- SLC17A9 expression is an independent predictor of overall survival and recurrence-free survival in GC patients.

- High SLC17A9 expression may be a valuable independent biomarker to predict poor prognosis for GC patients.

- SLC17A9 mRNA expression correlates positively with TP53 mutation.

Acknowledgments

The authors thank E Zhai and J Liao for their help in this study.

Financial \& competing interests disclosure

This study was supported by grants from the National Natural Science Foundation of China (81772579), '3\&3' Project of The First Affiliated Hospital of Sun Yat-sen University (Yulong He), and the Guangzhou Science and Technology Plan Projects (Health Medical Collaborative Innovation Program of Guangzhou; 201803040019). The authors have no other relevant affiliations or financial involvement with any organization or entity with a financial interest in or financial conflict with the subject matter or materials discussed in the manuscript apart from those disclosed.

No writing assistance was utilized in the production of this manuscript.

Ethical conduct of research

Ethical approval was obtained by the First Affiliated Hospital, Sun Yat-sen University and the authors have followed the principles outlined in the Declaration of Helsinki for all human or animal experimental investigations. In addition, for investigations involving human subjects, informed consent has been obtained from the participants involved.

Open access

This work is licensed under the Attribution-NonCommercial-NoDerivatives 4.0 Unported License. To view a copy of this license, visit http://creativecommons.org/licenses/by-nc-nd/4.0/ 


\section{References}

Papers of special note have been highlighted as: $\bullet$ of interest

1. Torre LA, Bray F, Siegel RL, Ferlay J, Lortet-Tieulent J, Jemal A. Global cancer statistics, 2012. CA. Cancer J. Clin. 65(2), 87-108 (2015).

2. Bertuccio P, Chatenoud L, Levi F. Recent pattern in gastric cancer: a global overview. Int. J. Cancer 3(125), 666-673 (2010).

3. Casamayor M, Morlock R, Maeda H, Ajani J. Targeted literature review of the global burden of gastric cancer. Ecancermedicalscience 12, 883 (2018).

4. Vrana D, Matzenauer M, Neoral C et al. From tumor immunology to immunotherapy in gastric and esophageal cancer. Int. J. Mol. Sci. 20(1), E13 (2018).

5. Sreedharan S, Shaik JH, Olszewski PK, Levine AS, Schioth HB, Fredriksson R. Glutamate, aspartate and nucleotide transporters in the SLC17 family form four main phylogenetic clusters: evolution and tissue expression. Bmc Genomics 11, 17 (2010).

6. Sesma JI, Kreda SM, Okada SF et al. Vesicular nucleotide transporter regulates the nucleotide content in airway epithelial mucin granules. Am. J. Physiol. Cell Physiol. 304(10), C976-C984 (2013).

7. Oya M, Kitaguchi T, Yanagihara Y et al. Vesicular nucleotide transporter is involved in ATP storage of secretory lysosomes in astrocytes. Biochem. Biophys. Res. Commun. 438(1), 145-151 (2013).

8. Sawada K, Echigo N, Juge N et al. Identification of a vesicular nucleotide transporter. Proc. Natl Acad. Sci. USA 105(15), 5683-5686 (2008).

- First identification of SLC17A9 protein as a vesicular nucleotide transporter.

9. Cao Q, Zhao K, Zhong XZ et al. SLC17A9 protein functions as a lysosomal ATP transporter and regulates cell viability. J. Biol. Chem. 289(33), 23189-23199 (2014).

10. Chen B, Jiang L, Zhong ML et al. Identification of fusion genes and characterization of transcriptome features in T-cell acute lymphoblastic leukemia. Proc. Natl Acad. Sci. USA 115(2), 373-378 (2018).

11. Yang L, Chen Z, Xiong W et al. High expression of SLC17A9 correlates with poor prognosis in colorectal cancer. Hum. Pathol. 84, 62-70 (2018).

12. Zhai E, Liang W, Lin Y et al. HSP70/HSP90-organizing protein contributes to gastric cancer progression in an autocrine fashion and predicts poor survival in gastric cancer. Cell. Physiol. Biochem. 2(47), 879-892 (2018).

13. Chandrashekar DS, Bashel B, Balasubramanya $S$ et al. UALCAN: a portal for facilitating tumor subgroup gene expression and survival analyses. Neoplasia 19(8), 649-658 (2017).

14. Cui J, Chen Y, Chou WC et al. An integrated transcriptomic and computational analysis for biomarker identification in gastric cancer. Nucleic Acids Res. 39(4), 1197-1207 (2011).

15. Deng N, Goh LK, Wang H et al. A comprehensive survey of genomic alterations in gastric cancer reveals systematic patterns of molecular exclusivity and co-occurrence among distinct therapeutic targets. Gut 61(5), 673-684 (2012).

16. Wang Q, Wen YG, Li DP et al. Upregulated INHBA expression is associated with poor survival in gastric cancer. Med. Oncol. 29(1), 77-83 (2012).

17. Szasz AM, Lanczky A, Nagy A et al. Cross-validation of survival associated biomarkers in gastric cancer using transcriptomic data of 1,065 patients. Oncotarget 7(31), 49322-49333 (2016).

18. Vasaikar SV, Straub P, Wang J, Zhang B. LinkedOmics: analyzing multi-omics data within and across 32 cancer types. Nucleic Acids Res. 46(D1), D956-D963 (2018).

19. Haanes KA, Kowal JM, Arpino G et al. Role of vesicular nucleotide transporter VNUT (SLC17A9) in release of ATP from AR42J cells and mouse pancreatic acinar cells. Purinerg. Signal. 10(3), 431-440 (2014).

- Physiological action and molecular mechanism of SLC17A9 in pancreatic ducts.

20. Harada Y, Kato Y, Miyaji T, Omote H, Moriyama Y, Hiasa M. Vesicular nucleotide transporter mediates ATP release and migration in neutrophils. J. Biol. Chem. 293(10), 3770-3779 (2018).

21. Hongzhou C, Longnian L, Wenjun W et al. Exome sequencing identifies SLC17A9 pathogenic gene in two Chinese pedigrees with disseminated superficial actinic porokeratosis. J. Med. Genet. 51(10), 699-704 (2014).

22. Erina T, Mitsutoshi T, Hitoshi H, Keisuke S, Yoshinori M, Shuji K. Autocrine regulation of TGF- $\beta 1$-induced cell migration by exocytosis of ATP and activation of P2 receptors in human lung cancer cells. J. Cell Sci. 125(Pt 21), 5051-5060 (2012).

- $\quad$ SLC17A9-dependent ATP release regulates TGF- $\beta 1$-induced migration and actin remodeling in human lung cancer cells.

23. Icard P, Fournel L, Wu Z, Alifano M, Lincet H. Interconnection between metabolism and cell cycle in cancer. Trends Biochem. Sci. 44(6), 490-501 (2019).

24. Lincet H, Icard P. How do glycolytic enzymes favour cancer cell proliferation by nonmetabolic functions? Oncogene 34(29), 3751-3759 (2015).

25. Lu Z, Hunter T. Metabolic kinases moonlighting as protein kinases. Trends Biochem. Sci. 43(4), 301-310 (2018). 
26. Dou Y, Wu HJ, Li HQ et al. Microglial migration mediated by ATP-induced ATP release from lysosomes. Cell Res. 22(6), 1022-1033 (2012).

27. Jang HH. Regulation of protein degradation by proteasomes in cancer. J. Cancer Prev. 4(23), 153-161 (2018).

28. Kolch W, Pitt A. Functional proteomics to dissect tyrosine kinase signalling pathways in cancer. Nat. Rev. Cancer 10(9), 618-629 (2010).

29. Gallo LH, Ko J, Donoghue DJ. The importance of regulatory ubiquitination in cancer and metastasis. Cell Cycle 16(7), 634-648 (2017).

30. Gurpinar E, Vousden KH. Hitting cancers' weak spots: vulnerabilities imposed by p53 mutation. Trends Cell Biol. 25(8), 486-495 (2015). 